\title{
Estimate Heterosis for Yield and Yield Components in Linseed (Linum usitatissimum L.) Germplasm
}

\author{
Ajeet Kumar ${ }^{1 *}$, Jitendra Kumar², Kushal Pal Singh Tomar ${ }^{1}$ and \\ Pradeep Kumar Sharma ${ }^{2}$
}
${ }^{1}$ Department of Plant Breeding and Genetics, SVP University of Agriculture and Technology, Meerut, India
${ }^{2}$ Department of Genetics and Plant Breeding, CCS University, Meerut, India

*Corresponding author

\begin{abstract}
A B S T R A C T
Keywords

Linseed (Linum usitatissimum L.), Heterosis,

Harvest index

Article Info

Accepted:

15 January 2018

Available Online:

10 February 2018

The present investigation entitled "To the estimate heterosis for yield and yield components in linseed (Linum usitatissimum L.) germplasm" was undertaken to collect information on genetic parameters for yield and its components from a ten parents diallel mating design in linseed at Crop Research Centre (Chirodi), Sardar Vallabhbhai Patel University of Agriculture \& Technology, Meerut (U.P.) during rabi 2013-14 and 2014-15. The experimental material was comprised of 10 diverse parents and their $45 \mathrm{~F}_{1} \mathrm{~s}$ excluding reciprocals of a diallel cross. All genotypes were evaluated in a Complete Randomized Block Design with three replications. The data were recorded on twelve characters; days to $50 \%$ flowering, days to maturity, plant height, number of primary branches per plant, number of secondary branches per plant, number of capsules per plant, number of seeds per capsule, 1000 seed weight, biological yield per plant, harvest index, oil content and seed yield per plant. Thirteen cross combinations which showed the highest value of heterosis for seed yield, namely; Sweta $\times$ Garima, Sweta $\times$ Shekhar, Sweta $\times$ Kiran, Sweta $\times$ Surbhi, Sweta $\times$ Mukta, Garima $\times$ Shekhar, Garima $\times$ Shubhra, Garima $\times$ Kiran, Garima x T-397, Garima $\times$ Neelam, Garima $\times$ Mukta, Garima $\times$ Hira and Shubhra $\times$ Neelam over better parent may be exploited for developing hybrids with better yield in linseed.
\end{abstract}

\section{Introduction}

Linseed (Linum usitatissimum L.) belongs to the genus Linum of the family Linaceae. The somatic chromosome number of the cultivated species is $2 n=30$ is cultivated for the main products, fiber (flax fiber) and seed oil (linseed) or both (dual purpose linseed), but recently it has gained a new interest in the emerging market of functional food due to its high content of fatty acids, alpha linolenic acid (ALA), an essential Omega-3 fatty acid and lignin oligomers which constitute about $57 \%$ of total fatty acids in linseed. Traditionally, the oil pressed from the seed (linseed oil) has been used for a variety of industrial purposes and the oil free meal could be fed to livestock. Omega-3 fatty acids lower levels of triglycerides in the blood, thereby reducing heart disease, and also show promise in the 
battle against inflammatory diseases such as rheumatoid arthritis. Linolenic acid (LA), an Omega-6 essential fatty acid is also found in linseed. Linseed oil contains three times as much Omega-3 fatty acid fatty than Omega-6 fatty acid.

The commercial exploitation of heterosis led to the remarkable yield advances in several cross pollinated crops. In self-pollinated crops, it is now well recognized that heterosis is very useful increasing productivity. The magnitude of heterosis provides a basis for genetic diversity and guideline to the choice of desirable parents for developing superior F1 hybrids so as to exploit hybrid vigour and for building gene pool to be exploitation in population improvement. In the present studies heterosis (mid-parent and betterparent) was estimated for seed yield and some important agronomic traits in $\mathrm{F} 1$ generation of linseed genotypes using diallelmodel suggested by Kempthorne (1961).

\section{Materials and Methods}

The experimental material included in present study comprised of $45 \mathrm{~F} 1$ 's and 10 parents were grown in a randomized block design with three replications. The experimental at Crop Research Centre of Sardar Vallabhbhai Patel University of Agriculture \& Technology, Meerut. The basic experimental material for this investigation comprised ten genotypes of linseed (Linum usitatissimum L.) namely Sweta, Garima, Shekhar, Shubhra, Kiran, Surbhi, T 397, Neelam, Mukta and Hira selected on the basis of morphological diversity for different agronomical traits. All possible single cross was made under $10 \times 10$ diallel mating system set excluding reciprocals. The data was subjected to analysis of variance according to Panse and Sukhatme (1961) the percent increase (+) or decrease (-) of $\mathrm{F} 1$ cross over mid parent as well as better parent was calculated to observe heterotic effects of all the parameters. To estimation of heterosis over the mid-parent and better-parent was calculated using the procedure of Shull, 1908 and (Fonseca and Patterson, 1968) respectively. The difference of $\mathrm{F} 1$ mean from the respective mid-parent and better-parent values was evaluated by using t-test according to Wynne et al., (1970).

Heterosis expressed as percentage increase or decrease of $\mathrm{F}_{1} \mathrm{~s}$ over better parent and economic parent was calculated by using following formula:

Heterosis $(\%)$ over better parent $=$
$\frac{\overline{\mathrm{F}}_{1}-\overline{\mathrm{BP}}}{\overline{B P}} \times 100$

Heterosis (\%) over mid parent = $\frac{\overline{\mathrm{F}}_{1}-\overline{\mathrm{MP}}}{\overline{M P}} \times 100$

Where,

$\bar{F}=$ mean value of $\mathrm{F} 1$

$\overline{B P}=$ mean value of better parent

$\overline{M P}=$ mean value of mid parent

\section{Results and Discussion}

The mean value for fourteen characters of $F_{1}$ hybrids were compared with the mid parent and better parent. Estimates of heterosis over better parent (heterobeltiosis) expressed as percentage increase or decrease are presented in Table-1 and described character wise as under:

\section{Days to $50 \%$ flowering}

Heterobeltiosis for days to $50 \%$ flowering ranged from -11.228 (Sweta $\times$ Hira) to 24.159 (Garima $\times$ Kiran). Ten crosses showed negative significant heterosis over better- 
parent heterosis. Similarly early flowering in linseed can provide adequate time for grain formation process and can certainly cause early maturity higher yields; therefore, negative heterosis is desirable for flowering. The data presented in Table 1 for $50 \%$ flowering showed that out of 45 crosses, Sweta $\times$ T-397 (-9.002) exhibited significant earliness for this character followed by Sweta $\times$ Mukta showed significant heterosis over mid-parent. Nine crosses showed significant negative heterosis over mid-parent heterosis.

\section{Days to maturity}

Heterobeltiosis ranged from -7.534 (Sweta $\times$ Shekhar) to 12.074 (Sweta $\times$ Shubhra). Out of 45 crosses 9 showed positive better-parent heterosis. Maximum heterosis over betterparent was observed in Sweta $\times$ Shubhra. Early maturity is useful in most of the plant species, especially linseed where delayed maturity causes losses to yield and quality of oil due to rise in temperature; therefore, negative heterosis is desirable for early maturity. Heterotic data presented in Table 1 showed that out of 45 crosses, 8 crosses showed significant negative heterosis over mid-parent.

\section{Plant height}

Heterobeltiosis for plant height values ranged from -10.048 (Sweta $\times$ Kiran) to 58.019 (Kiran $\times$ Mukta). Three crosses revealed negative significant heterosis over better parent for dwarf stature.Crosses Shubhra $\times$ Hira and Sweta $\times$ Neelam showed significant decrease in plant height over mid-parent. Three crosses showed significant negative heterosis and 13 crosses showed significant positive heterosis for plant height. Heterosis estimates over mid-parent (Table 1) showed that out of 45 crosses, 16 crosses had positive effects where values ranged from -32.928 $($ Shekhar $\times$ Shubhra) to 68.441 (Garima $\times$
Surbhi). Sixteen crosses showed positive significant heterosis over mid-parent. Betterparent heterosis ranged from -37.107 to 70.970 percent in crosses Shekhar $\times$ Kiran and Garima $\times$ Surbhi. The positive and significant heterosis over better parent, suggested that good performance for number of primary branches per plant.

\section{Number of secondary branches per plant}

Heterobeltiosis over the better parental ranged from -29.727 (Garima $\times$ T-397) to 48.688 $($ Kiran $\times$ Surbhi). Fifteen crosses were observed positive and significant heterosisover better parent (Table-I) for a number of secondary branches per plant. Heterosis estimates over mid-parent (Table I) showed that out of 45 crosses, 15 crosses had positive effects where values ranged from -29.227 $($ Surbhi $\times$ T-397) to 40.109 (Shubhra $\times$ Neelam).

\section{Number of capsules perplant}

Highest positive significant heterosis for more number of capsules per plant over betterparent was observed in crosses Garima $\times$ Kiran (46.993) followed by Garima $\times$ Shubhra (40.788) and Shekhar $\times$ T-397 (40.650). The maximum heterosis for number of capsules per plant over mid-parent (Table 1) was showed by Garima $\times$ Kiran (45.205) and Garima $\times$ Shubhra (43.700).

\section{Number of seeds per capsules}

Heterobeltiosis for number of seeds per capsules over better parental values ranged from -21.254 (Garima $\times$ Hira) to 31.265 (Shubhra $\times$ Kiran). Out of forty five crosses, only twelve best hybrids observed significant and positive heterosis over better parent (Table 1) which indicated that good performance for more number of seeds per capsule. 
Table.1 Estimation Heterosis of crosses for yield components in linseed (Linum usitatissimum L.)

\begin{tabular}{|c|c|c|c|c|c|c|}
\hline Characters & Days to $50^{c}$ & lowering & Days to ma & & Plant height & \\
\hline Crosses & MP & BP & MP & BP & MP & BP \\
\hline Sweta $\times$ Garima & -5.625 & $-5.779 * *$ & -1.610 & $-6.066^{* *}$ & $10.302 * *$ & 11.403 \\
\hline Sweta $\times$ Shekhar & $4.143 * *$ & $4.633 * *$ & $-4.934 * *$ & $-7.534 * *$ & 2.566 & 2.414 \\
\hline Sweta $\times$ Shubhra & $19.367 * *$ & $22.428 * *$ & $11.915 * *$ & $-12.074 * *$ & 1.719 & 1.786 \\
\hline Sweta $\times$ Kiran & $20.228 * *$ & $21.311 * *$ & 4.831 & 2.086 & $10.929 * *$ & $-10.048 * *$ \\
\hline Sweta $\times$ Surbhi & $9.267 * *$ & $10.290 * *$ & 0.010 & 1.041 & 1.934 & 2.087 \\
\hline Sweta $\times$ T-397 & $-9.002 * *$ & $-11.155^{* *}$ & $-3.076 * *$ & $-4.547 * *$ & $6.539 * *$ & 7.720 \\
\hline Sweta $\times$ Neelam & $-3.360 * *$ & $-4.698 * *$ & $3.256 * *$ & $-3.674 * *$ & $-7.6 .4^{*}$ & $-9.099 * *$ \\
\hline Sweta $\times$ Mukta & $-8.673 * *$ & $-10.616^{* *}$ & $4.311 * *$ & $7.944 * *$ & -4.061 & 9.579 \\
\hline Sweta $\times$ Hira & $-7.589 * *$ & $-11.228 * *$ & $-5.639 * *$ & $-6.101 * *$ & 8.399 & 10.869 \\
\hline Garima $\times$ Shekhar & $3.023 * *$ & $3.066 * *$ & 0.700 & -1.211 & -0.856 & -1.820 \\
\hline Garima $\times$ Shubhra & $12.343 * *$ & $12.630 * *$ & 2.790 & 7.033 & $13.075^{* *}$ & $13.731 * *$ \\
\hline Garima $\times$ Kiran & $21.857 * *$ & $24.159 * *$ & 3.669 & 1.579 & 0.742 & 0.734 \\
\hline Garima $\times$ Surbhi & 0.581 & 0.301 & 0.955 & $-2.632 *$ & 1.443 & 5.710 \\
\hline Garima $\times$ T-397 & $-7.523 * *$ & $-9.326 * *$ & -0.419 & $-6.303 * *$ & 1.173 & -1.359 \\
\hline Garima $\times$ Neelam & 1.145 & 1.408 & 3.268 & 1.000 & 5.577 & 6.676 \\
\hline Garima $\times$ Mukta & 0.436 & $0.761 * *$ & $6.666^{* *}$ & $5.821^{* *}$ & $4.521 * *$ & $5.715^{* *}$ \\
\hline Garima $\times$ Hira & $14.505^{* *}$ & $14.745^{* *}$ & -1.075 & -5.995 & $18.850 * *$ & $35.990 * *$ \\
\hline Shekhar $\times$ Shubhra & $-7.997 * *$ & $-8.840 * *$ & 1.124 & -1.468 & $-1.175 * *$ & $-2.266 * *$ \\
\hline Shekhar $\times$ Kiran & 0.194 & 0.077 & 1.302 & 1.178 & $6.635^{* *}$ & $7.389 * *$ \\
\hline Shekhar $\times$ Surbhi & $-2.099 * *$ & $-3.383 * *$ & 4.663 & 4.862 & 2.782 & 4.158 \\
\hline Shekhar $\times$ T-397 & -2.568 & -2.614 & -1.392 & $-5.503 * *$ & -1.321 & -1.458 \\
\hline Shekhar $\times$ Neelam & 0.009 & 0.719 & -2.634 & -4.895 & -1.060 & -2.148 \\
\hline Shekhar $\times$ Mukta & 1.803 & 1.756 & $9.747 * *$ & $8.514^{* *}$ & 0.291 & 0.755 \\
\hline Shekhar $\times$ Hira & $9.267 * *$ & $14.855^{* *}$ & 2.728 & 3.556 & 6.018 & 6.610 \\
\hline Shubhra $\times$ Kiran & 5.588 & 5.255 & -1.211 & -3.628 & -3.006 & $16.230 * *$ \\
\hline Shubhra $\times$ Surbhi & -2.703 & -7.411 & -1.106 & -1.970 & 0.262 & 0.446 \\
\hline Shubhra $\times$ T-397 & -5.257 & -10.925 & $4.641 * *$ & $6.255 * *$ & -0.839 & $9.704 * *$ \\
\hline Shubhra $\times$ Neelam & -4.02 & -6.408 & $-4.838 * *$ & $-5.080 * *$ & $8.304 * *$ & -0.233 \\
\hline Shubhra $\times$ Mukta & $-3.313 * *$ & $-4.999 * *$ & 3.828 & 0.059 & $7.033 * *$ & $14.406 * *$ \\
\hline Shubhra $\times$ Hira & $12.901 * *$ & $13.035^{* *}$ & 1.351 & 0.674 & $-15.794 * *$ & $26.726 * *$ \\
\hline Kiran $\times$ Surbhi & $6.268^{* *}$ & $6.019 * *$ & 1.561 & -0.065 & 1.537 & 2.535 \\
\hline Kiran $\times$ T-397 & 0.258 & 0.782 & 0.195 & 3.869 & 0.656 & 0.384 \\
\hline Kiran $\times$ Neelam & 2.012 & 3.747 & -0.533 & -2.726 & 8.535 & 1.123 \\
\hline Kiran $\times$ Mukta & 3.053 & 3.478 & 3.964 & 2.672 & $49.509 * *$ & $58.019 * *$ \\
\hline Kiran $\times$ Hira & $8.964 * *$ & $13.687 * *$ & 3.568 & 3.376 & 0.905 & 0.697 \\
\hline Surbhi $\times$ T-397 & 0.019 & 1.247 & $2.925^{* *}$ & $-5.386 * *$ & 9.652 & 2.399 \\
\hline Surbhi $\times$ Neelam & -1.054 & -3.509 & 1.950 & 1.315 & 5.539 & 7.592 \\
\hline Surbhi $\times$ Mukta & $-4.390 * *$ & $-7.457 * *$ & 5.609 & 2.647 & -2.929 & -2.180 \\
\hline Surbhi $\times$ Hira & $9.058 * *$ & $13.584 * *$ & $-3.770 * *$ & $-5.242 * *$ & $15.182 * *$ & $35.218 * *$ \\
\hline T-397 × Neelam & -3.310 & -6.873 & $-2.955^{* *}$ & $-4.834 * *$ & $10.535 * *$ & $11.658 * *$ \\
\hline T-397 $\times$ Mukta & -0.413 & -4.787 & 1.117 & -4.140 & -2.492 & -3.662 \\
\hline T-397 $\times$ Hira & 10.368 & $15.851^{* *}$ & $7.504 * *$ & $8.464^{* *}$ & $22.566 * *$ & 37.743 \\
\hline Neelam $\times$ Mukta & -4.101 & -4.833 & 5.097 & 1.530 & 1.389 & 1.331 \\
\hline Neelam $\times$ Hira & $13.994 * *$ & $16.253 * *$ & 3.087 & 2.141 & $20.861 * *$ & 35.753 \\
\hline Mukta $\times$ Hira & 14.492 & $16.109 * *$ & 2.067 & -2.270 & $19.432 * *$ & $23.086 * *$ \\
\hline
\end{tabular}


Contd...

\begin{tabular}{|c|c|c|c|c|c|c|}
\hline \multirow{2}{*}{$\begin{array}{l}\text { Characters } \\
\text { Crosses }\end{array}$} & \multicolumn{2}{|c|}{$\begin{array}{c}\text { Number Primary branches per } \\
\text { plant }\end{array}$} & \multicolumn{2}{|c|}{$\begin{array}{l}\text { Number secondary } \\
\text { branches per plant }\end{array}$} & \multicolumn{2}{|c|}{$\begin{array}{c}\text { Number of capsules per } \\
\text { plant }\end{array}$} \\
\hline & MP & BP & MP & BP & MP & BP \\
\hline Sweta $\times$ Garima & 1.720 & 2.842 & $8.732 * *$ & $9.048^{* *}$ & 0.598 & 0.543 \\
\hline Sweta $\times$ Shekhar & $0.570 * *$ & 1.142 & 6.880 & 7.837 & $17.962 * *$ & $29.078 * *$ \\
\hline Sweta $\times$ Shubhra & 1.554 & 2.791 & 0.180 & 0.244 & $21.379 * *$ & $22.270 * *$ \\
\hline Sweta $\times$ Kiran & $49.087 * *$ & $50.337 * *$ & 1.145 & 2.947 & $17.236 * *$ & $19.457 * *$ \\
\hline Sweta $\times$ Surbhi & $36.247 * *$ & $41.097 * *$ & 1.088 & 12.294 & 2.570 & 1.409 \\
\hline Sweta $\times$ T-397 & -3.728 & -4.197 & $-6.155^{* *}$ & $-7.085 * *$ & 1.807 & 1.612 \\
\hline Sweta $\times$ Neelam & $-6.658 * *$ & $-6.943 * *$ & $26.658 * *$ & $32.887 * *$ & $-27.732 * *$ & $36.887 * *$ \\
\hline Sweta $\times$ Mukta & $24.761 * *$ & $25.645^{* *}$ & 2.703 & 5.949 & 2.636 & $8.356 * *$ \\
\hline Sweta $\times$ Hira & -1.609 & -1.707 & 7.668 & 8.782 & 1.583 & 2.805 \\
\hline Garima $\times$ Shekhar & $58.286 * *$ & $64.683 * *$ & $9.800 * *$ & $8.415^{* *}$ & $-3.629 * *$ & $-4.419 * *$ \\
\hline Garima $\times$ Shubhra & 0.412 & 8.721 & 6.475 & $-7.744^{*}$ & $43.700 * *$ & $40.788 * *$ \\
\hline Garima × Kiran & $-4.885 * *$ & $-5.162 * *$ & -1.287 & -1.179 & $45.205 * *$ & $46.993 * *$ \\
\hline Garima $\times$ Surbhi & $68.441 * *$ & $70.970 * *$ & 6.694 & 7.289 & -0.903 & 0.118 \\
\hline Garima $\times$ T-397 & 1.920 & 2.207 & $-18.499 * *$ & $-29.727 * *$ & $37.845^{* *}$ & $26.582 * *$ \\
\hline Garima $\times$ Neelam & $40.687 * *$ & $50.805^{* *}$ & 7.894 & 8.060 & 2.754 & $-11.133 * *$ \\
\hline Garima × Mukta & 5.701 & -6.375 & 1.579 & 3.477 & -0.174 & 0.957 \\
\hline Garima $\times$ Hira & 7.277 & 9.560 & $-8.518 * *$ & $-10.806^{* *}$ & $-8.389 * *$ & $-17.203 * *$ \\
\hline Shekhar $\times$ Shubhra & $-32.928 * *$ & $38.558 * *$ & $-14.190 * *$ & $-17.870 * *$ & 13.544 & $24.672 * *$ \\
\hline Shekhar $\times$ Kiran & $-30.276^{* *}$ & -37.107 & $-8.595 * *$ & $-10.589 * *$ & $18.377 * *$ & $31.355^{* *}$ \\
\hline Shekhar $\times$ Surbhi & 2.402 & 4.983 & $-12.221 * *$ & $22.886^{* *}$ & $26.368 * *$ & $39.978 * *$ \\
\hline Shekhar × T-397 & $31.315^{* *}$ & $35.566 * *$ & $32.759 * *$ & $41.391 * *$ & $25.629 * *$ & $40.650 * *$ \\
\hline Shekhar $\times$ Neelam & $-0.891 * *$ & $-1.103 * *$ & 0.805 & 1.021 & $-10.624 * *$ & $-11.680 * *$ \\
\hline Shekhar $\times$ Mukta & $26.365 * *$ & $38.558 * *$ & $14.729 * *$ & 2.446 & 4.107 & 7.337 \\
\hline Shekhar $\times$ Hira & 5.017 & 4.045 & -3.497 & -8.242 & $-8.429 * *$ & $-13.820 * *$ \\
\hline Shubhra $\times$ Kiran & $37.315^{* *}$ & $38.372 * *$ & $-2.880 * *$ & -3.315 & 3.790 & 7.713 \\
\hline Shubhra $\times$ Surbhi & $34.059 * *$ & $41.860 * *$ & $17.474 * *$ & $28.717 * *$ & -2.757 & -1.107 \\
\hline Shubhra $\times$ T-397 & $-2.472 * *$ & $-3.566 * *$ & 2.402 & 3.143 & -4.116 & -4.297 \\
\hline Shubhra $\times$ Neelam & $34.788 * *$ & $41.609 * *$ & $40.901 * *$ & $42.448 * *$ & 0.710 & 1.784 \\
\hline Shubhra $\times$ Mukta & 1.238 & 2.791 & 8.342 & 12.963 & 0.541 & 1.073 \\
\hline Shubhra $\times$ Hira & -3.140 & -5.581 & 2.880 & 8.593 & -0.634 & -1.807 \\
\hline Kiran $\times$ Surbhi & $-28.739 * *$ & $36.221 * *$ & $42.718 * *$ & $48.688 * *$ & 0.358 & 3.445 \\
\hline Kiran $\times$ T-397 & 0.931 & 1.055 & $36.216 * *$ & $43.323 * *$ & $-20.882 * *$ & $-25.869 * *$ \\
\hline Kiran $\times$ Neelam & $29.111 * *$ & $37.471 * *$ & 1.916 & -2.762 & $15.299 * *$ & -2.082 \\
\hline Kiran $\times$ Mukta & 7.021 & -7.341 & $11.450 * *$ & -2.394 & -0.886 & 0.315 \\
\hline Kiran $\times$ Hira & 1.644 & 1.930 & $-14.500 * *$ & $-16.943 * *$ & 4.349 & 6.493 \\
\hline Surbhi $\times$ T-397 & $-9.586 * *$ & $-9.631 * *$ & $-29.227 * *$ & $-29.870 * *$ & -3.018 & -5.654 \\
\hline Surbhi $\times$ Neelam & $20.298 * *$ & $36.092 * *$ & $22.336 * *$ & $31.633 * *$ & 2.017 & 2.862 \\
\hline Surbhi $\times$ Mukta & -7.140 & -9.269 & $29.386^{* *}$ & $35.714 * *$ & -1.715 & -1.365 \\
\hline Surbhi $\times$ Hira & -2.504 & -6.984 & $-26.069 * *$ & $41.399 * *$ & -1.284 & -2.033 \\
\hline T-397 $\times$ Neelam & $-17.953 * *$ & $-21.153 * *$ & 0.120 & $-22.427 * *$ & $25.83^{* *}$ & $40.276^{* *}$ \\
\hline T-397 $\times$ Mukta & 6.939 & 7.544 & -7.662 & $-26.864 * *$ & $-19.098 * *$ & $33.697 * *$ \\
\hline T-397 $\times$ Hira & -7.374 & -8.566 & $21.832 * *$ & $38.457 * *$ & $-20.711 * *$ & $33.572 * *$ \\
\hline Neelam $\times$ Mukta & $-13.514 * *$ & $-3.471 * *$ & $35.336 * *$ & $31.340 * *$ & 2.300 & 2.942 \\
\hline Neelam $\times$ Hira & $26.404 * *$ & $-47.586 * *$ & -6.047 & -8.095 & -0.020 & -4.839 \\
\hline Mukta $\times$ Hira & $33.122 * *$ & $29.897 * *$ & -1.927 & -2.720 & 0.555 & -2.163 \\
\hline
\end{tabular}


Contd...

\begin{tabular}{|l|}
\hline Characters \\
\hline Crosses \\
\hline Sweta $\times$ Garima \\
\hline Sweta $\times$ Shekhar \\
\hline Sweta $\times$ Shubhra \\
\hline Sweta $\times$ Kiran \\
\hline Sweta $\times$ Surbhi \\
\hline Sweta $\times$ T-397 \\
\hline Sweta $\times$ Neelum \\
\hline Sweta $\times$ Mukta \\
\hline Sweta $\times$ Hira \\
\hline Garima $\times$ Shekhar \\
\hline Garima $\times$ Shubhra \\
\hline Garima $\times$ Kiran \\
\hline Garima $\times$ Surbhi \\
\hline Garima $\times$ T-397 \\
\hline Garima $\times$ Neelam \\
\hline Garima $\times$ Mukta \\
\hline Garima $\times$ Hira \\
\hline Shekhar $\times$ Shubhra \\
\hline Shekhar $\times$ Kiran \\
\hline Shekhar $\times$ Surbhi \\
\hline Shekhar $\times$ T-397 \\
\hline Shekhar $\times$ Neelam \\
\hline Shekhar $\times$ Mukta \\
\hline Shekhar $\times$ Hira \\
\hline Shubhra $\times$ Kiran \\
\hline Shubhra $\times$ Surbhi \\
\hline Shubhra $\times$ T-397 \\
\hline Shubhra $\times$ Neelam \\
\hline Shubhra $\times$ Mukta \\
\hline Shubhra $\times$ Hira \\
\hline Kiran $\times$ Surbhi \\
\hline Kiran $\times$ T-397 \\
\hline Kiran $\times$ Neelam \\
\hline Kiran $\times$ Mukta \\
\hline Kiran $\times$ Hira \\
\hline Surbhi $\times$ T-397 \\
\hline Surbhi $\times$ Neelam \\
\hline Surbhi $\times$ Mukta \\
\hline Surbhi $\times$ Hira \\
\hline T-397 $\times$ Neelam \\
\hline T-397 $\times$ Mukta \\
\hline T-397 $\times$ Hira \\
\hline Neelam $\times$ Mukta \\
\hline Neelam $\times$ Hira \\
\hline Mukta $\times$ Hira \\
\hline
\end{tabular}

\begin{tabular}{|c|c|c|c|c|c|}
\hline \multicolumn{2}{|c|}{ Number of seeds per capsule } & \multicolumn{2}{|c|}{1000 seed weight $(\mathrm{g})$} & \multicolumn{2}{|c|}{$\begin{array}{c}\text { Biological yield per plant } \\
(\mathrm{g})\end{array}$} \\
\hline MP & BP & MP & BP & MP & BP \\
\hline 2.088 & 4.815 & $13.446^{* *}$ & $16.806^{* *}$ & $29.590 * *$ & $34.342 * *$ \\
\hline$-19.175 * *$ & $-21.111 * *$ & 0.671 & $0.986 * *$ & $29.112 * *$ & $30.559 * *$ \\
\hline 0.122 & 0.889 & $13.474 * *$ & $18.944 * *$ & 5.667 & 6.138 \\
\hline $15.364 * *$ & $17.778 * *$ & 5.647 & 6.503 & $16.114 * *$ & $18.540 * *$ \\
\hline $24.884^{* *}$ & $27.778 * *$ & $-19.555^{* *}$ & $19.915^{* *}$ & $39.174 * *$ & $39.906^{* *}$ \\
\hline$-8.645^{* *}$ & $-10.370 * *$ & 6.064 & 2.912 & 5.133 & 6.861 \\
\hline$-14.996 * *$ & $-16.296 * *$ & 1.738 & 2.913 & $-34.474 * *$ & $-36.503 * *$ \\
\hline 14.912 & 18.148 & $31.498 * *$ & $35.675 * *$ & 0.312 & 0.692 \\
\hline-7.361 & -10.105 & $32.144 * *$ & $37.323 * *$ & 0.696 & 1.364 \\
\hline 1.065 & 3.502 & 2.544 & 9.443 & $25.049 * *$ & $31.193 * *$ \\
\hline $14.106^{* *}$ & $14.106^{* *}$ & $20.671 * *$ & $24.424 * *$ & $27.606 * *$ & $38.374 * *$ \\
\hline 10.841 & 10.841 & $30.630 * *$ & $32.057 * *$ & 6.298 & 7.237 \\
\hline 0.321 & 0.321 & 1.006 & 5.484 & 1.861 & $1.202 * *$ \\
\hline 1.319 & 1.319 & 5.890 & 6.090 & $19.710 * *$ & $32.992 * *$ \\
\hline-5.556 & -5.556 & $16.258 * *$ & $16.031 * *$ & $26.904 * *$ & $29.347 * *$ \\
\hline $22.934 * *$ & $22.934 * *$ & 8.430 & 9.898 & $37.576^{* *}$ & $41.725 * *$ \\
\hline$-21.254 * *$ & $-21.254 * *$ & 10.630 & 11.623 & $23.033 * *$ & $38.822 * *$ \\
\hline$-24.514 * *$ & $24.514 * *$ & 10.009 & 11.311 & -9.295 & -10.797 \\
\hline 2.724 & 2.724 & $75.303 * *$ & $73.618 * *$ & 1.530 & 2.998 \\
\hline 8.949 & 8.949 & -4.179 & -5.788 & 4.163 & 5.823 \\
\hline$-14.165 * *$ & $-14.165^{* *}$ & -6.243 & -8.411 & -1.139 & -1.873 \\
\hline $25.292 * *$ & 25.292 & 0.069 & 1.719 & $-27.658 * *$ & $37.500 * *$ \\
\hline 8.560 & 8.560 & 6.243 & 12.648 & $27.816 * *$ & $35.635^{* *}$ \\
\hline $31.010 * *$ & $31.010 * *$ & 2.053 & 3.587 & 7.722 & 9.571 \\
\hline $31.265^{* *}$ & $31.265 * *$ & $38.684 * *$ & $48.513 * *$ & -0.095 & -1.945 \\
\hline 3.291 & 3.291 & $31.381 * *$ & $33.882 * *$ & -9.634 & -10.573 \\
\hline 5.617 & 5.617 & $31.579 * *$ & $33.305 * *$ & $5.982 *$ & 6.467 \\
\hline-2.965 & -2.965 & -2.705 & -4.315 & $-22.295^{* *}$ & $-31.898 * *$ \\
\hline 11.411 & 11.411 & $20.943 * *$ & $20.360 * *$ & 2.896 & 6.869 \\
\hline 6.132 & 6.132 & 10.380 & 11.469 & $-14.121 * *$ & $-14.661 * *$ \\
\hline$-12.804 * *$ & $-12.804 * *$ & $-15.855^{* * *}$ & $-16.373 * *$ & 10.077 & 11.400 \\
\hline $25.327 * *$ & $25.327 * *$ & -6.705 & -7.991 & $-11.807 * *$ & $-18.383^{* *}$ \\
\hline 5.378 & 5.378 & 15.789 & 10.095 & $28.937 * *$ & $34.525 * *$ \\
\hline 2.019 & 2.019 & -9.786 & -10.186 & $-29.756^{* *}$ & $-33.023 * *$ \\
\hline$-16.028 * *$ & $-16.028 * *$ & $-14.949 * *$ & $-14.538 * *$ & $-1.807 * *$ & 1.949 \\
\hline $25.327 * *$ & $25.327 * *$ & 0.704 & 2.523 & 3.810 & 4.312 \\
\hline 1.926 & 1.926 & -5.122 & -9.675 & $30.348 * *$ & $41.822 * *$ \\
\hline 9.149 & 9.149 & -6.366 & -9.832 & $-17.668 * *$ & $-20.519 * *$ \\
\hline$-18.815^{* *}$ & $-18.815^{* *}$ & 8.178 & -9.129 & -6.866 & -7.427 \\
\hline 22.633 & $22.633 * *$ & $22.077 * *$ & $27.634 * *$ & $-18.277 * *$ & $-29.837 * *$ \\
\hline $29.176^{* *}$ & 29.176 ** & 14.165 & 8.756 & 11.591 & $21.682 * *$ \\
\hline 8.892 & 8.892 & 0.700 & 1.878 & -2.572 & 4.293 \\
\hline 4.630 & 4.630 & $56.250 * *$ & $54.399 * *$ & $39.859 * *$ & $41.988 * *$ \\
\hline$-18.118 * *$ & $-18.118 * *$ & 9.257 & $-14.461 * *$ & 2.346 & 3.788 \\
\hline 1.725 & 1.725 & $38.403 * *$ & $31.952 * *$ & 4.003 & 5.403 \\
\hline
\end{tabular}


Contd...

\begin{tabular}{|c|c|c|c|c|c|c|}
\hline \multirow{2}{*}{$\begin{array}{l}\text { Characters } \\
\text { Crosses }\end{array}$} & \multicolumn{2}{|c|}{ Harvest index (\%) } & \multicolumn{2}{|c|}{ Oil content (\%) } & \multicolumn{2}{|c|}{ Seed yield per plant $(\mathrm{g})$} \\
\hline & MP & $\mathrm{BP}$ & MP & BP & MP & BP \\
\hline Sweta $\times$ Garima & 1.895 & 1.819 & -3.658 & -4.183 & $32.848 * *$ & $33.213^{* *}$ \\
\hline Sweta $\times$ Shekhar & 6.738 & 7.082 & 0.802 & 1.533 & $40.208 * *$ & $45.153 * *$ \\
\hline Sweta $\times$ Shubhra & 0.770 & 0.597 & 3.784 & 4.637 & -1.434 & -1.124 \\
\hline Sweta $\times$ Kiran & 1.837 & 3.697 & $-5.114 * *$ & $-7.936 * *$ & $31.681 * *$ & $39.287 * *$ \\
\hline Sweta $\times$ Surbhi & 1.104 & 2.879 & $-7.729 * *$ & $8.289 * *$ & $47.354 * *$ & $35.455^{* *}$ \\
\hline Sweta $\times$ T-397 & 5.599 & $11.280 * *$ & -0.659 & -2.824 & 1.006 & -0.070 \\
\hline Sweta $\times$ Neelum & 3.935 & 4.617 & -3.558 & -4.114 & -0.896 & -0.649 \\
\hline Sweta $\times$ Mukta & 3.092 & $15.254 * *$ & 1.348 & 1.972 & $34.105 * *$ & $41.176^{* *}$ \\
\hline Sweta $\times$ Hira & $-24.179 * *$ & $-26.296 * *$ & 0.682 & 2.778 & -1.321 & $25.246^{* *}$ \\
\hline Garima $\times$ Shekhar & -3.3 .99 & -4.801 & 0.072 & -2.723 & $20.653 * *$ & $32.575 * *$ \\
\hline Garima $\times$ Shubhra & -5.888 & $-11.391 * *$ & $13.310 * *$ & $16.566 * *$ & $32.510 * *$ & $45.422 * *$ \\
\hline Garima $\times$ Kiran & 8.937 & $14.862 * *$ & -2.526 & -4.919 & $23.915 * *$ & $36.043 * *$ \\
\hline Garima $\times$ Surbhi & -4.033 & -5.532 & $14.130 * *$ & $14.184 * *$ & 0.659 & $26.999 * *$ \\
\hline Garima $\times$ T-397 & 0.089 & $13.560 * *$ & 3.266 & 4.865 & $25.348 * *$ & $34.462 * *$ \\
\hline Garima $\times$ Neelam & 0.809 & 0.879 & 5.014 & 5.043 & $20.806^{* *}$ & $30.771 * *$ \\
\hline Garima $\times$ Mukta & $32.796 * *$ & $39.101 * *$ & $-5.953 * *$ & $-6.037 * *$ & $20.050 * *$ & $37.764 * *$ \\
\hline Garima $\times$ Hira & $-14.710^{* *}$ & $17.030 * *$ & 3.603 & $5.129 * *$ & $34.006^{* *}$ & $42.814^{* *}$ \\
\hline Shekhar $\times$ Shubhra & 0.894 & 0.203 & 0.408 & 0.444 & -3.034 & -0.727 \\
\hline Shekhar $\times$ Kiran & 2.062 & 5.671 & 0.209 & 0.147 & $8.842 * *$ & 0.418 \\
\hline Shekhar $\times$ Surbhi & 0.749 & 1.818 & -1.198 & -3.900 & $17.718 * *$ & 0.967 \\
\hline Shekhar $\times$ T-397 & -0.146 & -4.776 & $7.706^{* *}$ & $8.795^{* *}$ & -0.972 & -1.662 \\
\hline Shekhar $\times$ Neelam & $15.688 * *$ & $21.976 * *$ & -2.751 & -3.494 & -1.913 & -1.722 \\
\hline Shekhar $\times$ Mukta & 5.214 & 8.043 & -1.250 & -3.925 & -0.813 & $24.395 * *$ \\
\hline Shekhar $\times$ Hira & $-26.524 * *$ & $27.485 * *$ & $8.423^{* *}$ & $9.567 * *$ & $18.652 * *$ & $-20.515^{* *}$ \\
\hline Shubhra $\times$ Kiran & 7.216 & 8.401 & -2.643 & -3.974 & -0.256 & -0.929 \\
\hline Shubhra $\times$ Surbhi & $33.013 * *$ & $34.620 * *$ & 0.864 & 0.940 & 0.953 & $12.989 * *$ \\
\hline Shubhra $\times$ T-397 & $26.858 * *$ & $34.980 * *$ & 1.772 & 1.441 & $21.964 * *$ & $29.112 * *$ \\
\hline Shubhra $\times$ Neelam & -2.861 & -7.233 & 1.855 & 1.941 & $23.771 * *$ & $30.441 * *$ \\
\hline Shubhra $\times$ Mukta & 0.666 & 0.817 & 3.803 & 4.446 & $22.495 * *$ & $16.494 * *$ \\
\hline Shubhra $\times$ Hira & $15.227 * *$ & $17.634 * *$ & -1.541 & -3.750 & $22.581 * *$ & $28.690 * *$ \\
\hline Kiran $\times$ Surbhi & $-10.051 * *$ & 10.325 & $8.608 * *$ & $10.799 * *$ & 0.041 & -0.255 \\
\hline Kiran $\times$ T-397 & $-8.911 * *$ & $19.554 * *$ & $8.427 * *$ & $9.188 * *$ & $-18.440 * *$ & $-22.467 * *$ \\
\hline Kiran $\times$ Neelam & $26.964 * *$ & $22.124 * *$ & $10.619 * *$ & $12.787 * *$ & -1.239 & $-13.311 * *$ \\
\hline Kiran $\times$ Mukta & $28.695 * *$ & 11.922 & $8.439 * *$ & $10.610 * *$ & $-18.472 *$ & $-16.823 * *$ \\
\hline Kiran $\times$ Hira & 11.241 & 1.370 & 8.082 & $8.910 * *$ & -1.099 & -0.564 \\
\hline Surbhi $\times$ T-397 & 10.310 & 2.837 & 0.376 & 0.835 & $19.373 * *$ & 2.019 \\
\hline Surbhi $\times$ Neelam & $22.584 * *$ & $28.259 * *$ & $-5.421 * *$ & $-5.450 * *$ & $13.459 * *$ & $25.732 * *$ \\
\hline Surbhi $\times$ Mukta & $34.575^{* *}$ & $37.342 * *$ & 4.705 & 4.731 & $28.104 * *$ & $25.710 * *$ \\
\hline Surbhi $\times$ Hira & 1.450 & 2.260 & $-6.564 * *$ & $-7.986 * *$ & $-10.931 * *$ & $-22.899 * *$ \\
\hline T-397 $\times$ Neelam & 2.502 & 3.637 & $6.653 * *$ & $8.168 * *$ & -1.696 & -0.159 \\
\hline T-397 $\times$ Mukta & 0.157 & 1.066 & -3.092 & -4.609 & -1.670 & $14.611^{* *}$ \\
\hline T-397 $\times$ Hira & -0.873 & -0.961 & -0.505 & -0.577 & -0.131 & -0.589 \\
\hline Neelam $\times$ Mukta & $35.454 * *$ & $39.936 * *$ & -3.041 & -3.098 & -0.974 & $17.130^{* *}$ \\
\hline Neelam $\times$ Hira & 6.297 & $17.549 * *$ & -1.073 & -2.609 & -2.341 & -0.337 \\
\hline Mukta $\times$ Hira & $11.316^{* *}$ & $13.495 * *$ & -3.026 & -4.477 & $11.895^{* *}$ & -1.552 \\
\hline
\end{tabular}

* Significant at $5 \%$ probability level ** Significant at $1 \%$ probability level 
Over mid-parent heterosis varied from 19.165 (Sweta $\times$ Shekhar) to 27.206 (Shekhar $\times$ Hira). Whereas, nine hybrids had significant and negative heterosis over mid parent, indicated that poor performance for number of seeds per capsule.

\section{0 seed weight}

Heterobeltiosis for hybrids over their better parental values ranged from -16.373 (Kiran $\times$ Surbhi) to 73.618 (Shekhar $\times$ Kiran). For 1000 Seed weight fifteen crosses exhibited significant positive heterosis for 1000 Seed weight over mid-parent. Maximum positive heterosis over mid-parent was shown by Shekhar $\times$ Kiran (75.303) followed by Neelam $\times$ Mukta 25 (56.250) and Shubhra $x$ Kiran (38.684). Seventeen crosses showed positive heterosis over better-parent (Table1). Maximum positive heterosis for 1000 Seed weight over better-parent was recorded by cross Shekhar $\times$ Kiran (73.618) followed by Neelam $\times$ Mukta (54.399) and Shubhra $\times$ Kiran 5 (48.513). While three hybrids showed negative and significant heterosis over better parent for this character.

\section{Biological yield per plant}

Heterobeltiosis for biological yield per plant values ranged from $-36.503($ Sweta $\times$ Neelam) to 41.988 (Neelam $\times$ Mukta). Eighteen hybrids exhibited positive significant heterosis over better parental). For biological yield per plant sixteen crosses exhibited significant positive heterosis for biological yield per plant over mid-parent. Heterotic data presentedinTable1.

\section{Harvest index}

Heterobeltiosis ranged from -11.391 (Garima $\times$ Shubhra) to $39.936($ Neelam $\times$ Mukta). The hybrids viz., Nineteen crosses were observed positive significant heterosis over better parent for harvest index. Whereas, only one hybrid exhibited negative significant heterosis over better parent for this character. Over mid-parent heterosis ranged from -24.179 $($ Sweta $\times$ Hira) to 35.454 (Neelam $\times$ Mukta) . The 11 hybrids revealed positive significant heterosis over mid parent for this trait, whereas five hybrids showed negative significant heterosis over mid parent for harvest index in Table-1.

\section{Oil content}

Over better parent for oil content values ranged from -7.936 (Sweta $\times$ Kiran) to 16.566 (Garima $\times$ Shubhra). Out of forty five hybrids, only twelve hybrids were found positive significant heterosis over better parent for high oil content, while four hybrids showed significant negative heterosis over better parent for low oil content. Heterosis over mid parent varied from -7.729 (Sweta $\times$ Surbhi) to 14.130 (Garima $\times$ Surbhi). Ten crosses were exhibited significant positive heterosis over mid parent, whereas four hybrids observed significant negative heterosis over mid parent for oil content in Table1.

\section{Seed Yield per plant}

In the present study mid-parent heterosis for seed yield per plant ranged from -20.050 to 47.354 percent in crosses (Table 1) Garima $\times$ Mukta and Sweta $\times$ Surbhi respectively. Twenty one crosses exhibited positive significant mid-parent heterosis and twenty four crosses showed positive and significant heterosis over better parent for high seed yield per plant. Better-parent ranged from -22.899 $($ Surbhi $\times$ Hira) to 45.422 (Garima $\times$ Shubhra).

The Similar results on the importance of these traits have been reported by Galkin (1973), Patil and Chopde (1983), Dakhore and Narkhede (1987), Wang et al., (1990), Mishra 
and Rai (1993),), Saraswat and Kumar (1993),Verma and Sinha (1993), Yadav and Gupta (1999), Sharma et al., (2005), Vishnu et al., (2005), Singh et al., (2005), Mohammadi et al., (2010), Deepak et al., (2011), Yadav et al., (2013), Pali and Mehta (2014) and Kumar et al., (2014).Considering the predominance of non-additive gene action for yield and yield contributing traits manifesting high magnitude of hybrid vigor, heterosis breeding may be useful for improving yield in this crop.

Out of forty five crosses, only thirteen crosses exhibited superiority over better parent (heterobeltiosis) more than $10 \%$ for seed yield. The hybrids namely Sweta x Garima, Sweta x Shekhar, Sweta x Kiran, Sweta x Surbhi, Sweta x Mukta, Garima x Shekhar, Garima x Shubhra, Garima x Kiran, Garima x T-397, Garima x Neelam, Garima x Mukta, Garima x Hira and Shubhra x Neelam showed more than $30 \%$ heterobeltiosis over better parents. These individual crosses can be exploited in heterosis breeding programme for improvement in seed yield and also suggesting that it is possible to produce higher seed yield varieties.

\section{Acknowledgments}

All authors acknowledge the Head, Genetics and Plant Breeding, SVP University of Agriculture and Technology, Meerut provided the facilities and JK acknowledge the ICMR for the fellowship grant during this research.

\section{References}

Dakhore, S.R. and Narkhede, M.N. 1987. Heterosis in relation to combining ability effects in linseed (Linum usitatissimum L.) Maharashtra Agric. Uni. J. 12(3): 308-310.

Deepak, G.; Rao, S. S. and Pandagare, J. M. 2011. Genetic analysis of yield and yield attributing characters in linseed (Linum usitatissimum L.). Asian Journal of Bio Science 6(1):16-22.

Fonseca, S. and Patterson. F. 1968. Hybrid vigour in a seven parent diallel cross in common winter wheat. (Linum usitatissimum L.). Crop Sci.8: 85-88.

Galkin, F.M. 1973. Heterosis in inter-varietal hybrids of linseed. Byal Maunchtackhan Malichr Kulhrame, 2: 7-10.

Kempthorne, O., and Curnow, R.N. 1961. The partial diallel cross. Biometrics, 17(2): 229-250.

Kumar, S.; Kumar, R.; Kumar, S.; Singh, H.; Kumar, S. and Singh, M. P. (2014). Estimation of heterosis in linseed (Linum usitatissimum L.). J Agric Sci. 10(1):356-359.

Mishra, V.K. and Rai M. 1993. Estimates of heterosis for seed yield and oil linseed (Linum usitatissimum L.). Indian J. Genetics. 53 (2): 161-164.

Mohammadi, A. A.; Saeidi, G. and Arzani, A. 2010.Genetic analysis of some agronomic traits in flax (Linum usitatissimum L.). Australian Journal of Crop Science 4(5):343-352.

Pali, V. and Mehta, N. 2014. Combining ability and heterosis for seed yield and it's attributes in linseed (Linum $\begin{array}{ll}\text { usitatissimum } & \text { L.).The Bioscan }\end{array}$ 9(2):701-706.

Panse, V.G. and Sukhatme, P.V. 1961. Statistical methods for agricultural workers. ICAR, New Delhi $4^{\text {th }}$ edition 235-257.

Patil, V.D. and Chopde, P.R. 1983. Heterosis in relation to GCA and SCA effects in linseed. Indian J. Genet. 43 (2): 226228.

Saraswat, A.V. and Kumar, Satyendra 1993. Heterosis and inbreeding depression in some early hybrids of linseed. In heterosis breeding in crop plant theory and application symposium Ludhiana pp 23-24. 
Sharma, R.; Tiwari, S.K.; Singh, P. and Ramakant 2005. Heterobeltiosis and inbreeding depression in linseed. Agricultural Science Digest. 25(1): 3537.

Shull, G.H. 1908. The composition of field of maize, Rept. Am. Breeders Assoc. 4: 246-301

Singh, P.; Singh, D. and Singh, S.K. 2005. Heterosis in relation to other genetic parametersin linseed. Farm Science Journal, 14(2):1-3.

Verma, A.K. and Sinha, P.K. 1993. Heterosis in linseed. Theory and application: short communication: symposium Ludhiana 23-24.

Vishnu, A.; Shah, M.A. and Lakshyadeep. 2005. Studies on heterosis and combining ability for fatty acids in linseed (Linum usitatissimum L.). Indain J. Genetics. 63 (5):29-35.
Wang, YuFu; Yan Zhongfeng; Fan Jian Qiaogu angjun; Lu yun; Wu Guangwen; Wang Diankui; Yang Lijun and Wang Yan hua.1990. Study on heterosis and affinity of flax. Chinas Fibre Crops. No. 2: $10-13$

Wynne, J.C., D.A. Emery and P.M. Rice, 1970. Combining ability analysis over environments in spring wheat. Wheat In for. Serv. Japan. 67:21-24.

Yadav, P.; Verma, P. N.; Yadav, R. K. and Singh, S. 2013. Combining ability and heterosis analysis for seed yield and its related traits in linseed (Linum usitatissimum L.). Progressive Research 8:593-596.

Yadav, R.K. and Gupta, R.R. 1999. Genetic studies for some metric and quality characters in linseed under different environments. Crop Res. J. 18 (1): 5759.

\section{How to cite this article:}

Ajeet Kumar, Jitendra Kumar, Kushal Pal Singh Tomar and Pradeep Kumar Sharma. 2018. To the Estimate Heterosis for Yield and Yield Components in Linseed (Linum usitatissimum L.) Germplasm. Int.J.Curr.Microbiol.App.Sci. 7(02): 1602-1611. doi: https://doi.org/10.20546/ijcmas.2018.702.193 